\title{
Special issue on ICONIP 2012
}

\author{
Zhigang Zeng $\cdot$ Tingwen Huang $\cdot$ Chuandong $\mathbf{~ L i} \cdot$ \\ He Huang $\cdot$ Huiwei Wang
}

Published online: 4 December 2013

(C) Springer-Verlag London 2013

As one of the top conferences on intelligent computation, the 19th International Conference on Neural Information Processing (ICONIP 2012) was successfully held in Doha, Qatar, from November 11th to 15th, 2012, and covered wide topics on researches and applications of neural network in various fields. To continue such success, a special issue is launched on "Computational intelligence methods and Applications," with 19 high-quality papers selected from ICONIP 2012 in Neural Computing and Applications.

The purpose of this special issue is to track the latest process of researches and applications of neural networks, computational intelligence methods, and others. It is well known that computational intelligence as a hot focal will promote the development of society in the fields of neural computation, fuzzy computation, evolution computation, and so on. Therefore, it is meaningful to investigate the new theory, methodology, and tools for the computational intelligence study. To this end, the 19 selected papers in this special issue can be categorized into three major sections under this theme.

Z. Zeng

Huazhong University of Science and Technology, Wuhan, China

T. Huang ( $\square)$

Texas A\&M University at Qatar, Doha, Qatar

e-mail: tingwen.huang@qatar.tamu.edu

C. $\mathrm{Li}$

Southwest University, Chongqing, China

H. Huang

School of Electronics and Information Engineering,

Soochow University, Suzhou, China

H. Wang

College of Computer Science, Chongqing University,

Chongqing, China
The first section is focused on computational intelligence methods and applications in finance. Q.F. Meng et al. propose a multi-agent model to observe the impact of small-world network characteristics on incentive effects. They design two experiment scenarios to study the impact of the probability of replacement objects and the number of comparison objects on incentive effects. Experimental results show that under the impact of inequity aversion psychological, greater probability of replacement comparison objects will induce to lower profit and product sales volume of the manufacturer. D.H. Zhang et al. propose a supply disruption emergency management model of supply chain in the perspective of risk management. They also discuss whether the decision-making mechanism of the case-based reasoning can bring better effect for supply disruption based on numerical experiment, and a conclusion is reached that based on case-based reasoning, the emergency management mechanism can provide better management of the supply emergency disruption. Utilizing 157 samples of Chinese college-graduate village official, $\mathrm{L}$. Xiao and M. Fan conduct an empirical study and find out that the social network may not always promote entrepreneurial intention. S.P. Cheng et al. propose an estimation of distribution algorithm to solve the NP-hard problem in service selection and present a web service selection method based on the Univariate Marginal Distribution Algorithm (UMDA). Global service selection problem is modeled as an optimization problem. The characteristics of global service selection problem are not considered while adopting the basic UMDA algorithm to solve the problem.

The second section is focused on neural dynamics and dynamic systems. H. Wang et al. analyze the dynamical characteristics of a two-dimensional simplified $\mathrm{H}-\mathrm{H}$ model exposed to external electric fields. The necessary and sufficient condition for the existence of the Hopf bifurcation is 
derived. The dynamic behaviors, stability of equilibrium points, limit cycles, canards, as well as the bifurcation of the simplified and original models are extensively investigated. S.P. Fard and Z. Zainuddin investigate the universal approximation capability of a three-layered feedforward flexible approximate identity neural networks under the Lp[a; b]-norm. Y. Dai et al. work on the HP memristor and integrate the consideration of $R_{\text {on }}$, whose influence is usually eliminated by suggestion, and propose an expanded HP memristor whose memristance is easy to change under certain voltage load. This kind of memristor model is a good candidate for neural network, especially, in binary computation. C.J. Li et al. use Lyapunov-Ruzimiki method to study the global exponential synchronization of a class of discrete delayed complex networks with switching topology. The impulsive scheme is designed to work at the instant of switching occurrence. A time-varying delaydependent criterion for impulsive synchronization is given to ensure the delayed discrete complex networks switching topology tending to a synchronous state.

The third section is focused on practical applications of neural computing. A. Ciccazzo et al. show that the surrogate models given by the support vector machine can be applied in the design of real industrial circuits, offering a method of analyzing these circuits without resorting to a heavy use of costly circuit simulations. The SVM model is also compared to the surrogate models given by the response surface methodology (RSM) using commercial software currently adopted for this kind of applications. L.P. Dinu and R.T. Ionescu study several clustering methods based on rank distance. The K-means algorithm represents each cluster by a single mean vector. The mean vector is computed with respect to a distance measure. Two $\mathrm{K}$-means algorithms based on rank distance are described. H. Karimi et al. propose a general conductance model of DNA sensor based on grapheme. The current-voltage characteristic of nanostructured graphene-based DNA sensor is evaluated. By increasing the complementary DNA concentration, the drain current is going toward higher amounts. $\mathrm{X}$. Wu et al. propose a new Bayesian network method based on Gaussian assumptions, termed as Gaussian dynamic Bayesian network, to capture the temporal characteristics of connectivity with less associated loss of information. A set of synthetic data is generated to measure the robustness of this method. C. Lian et al. use a novel neural network technique called the ensemble of extreme learning machine (E-ELM) to investigate the interactions of different inducing factors affecting the evolution of landslide. Grey relation analysis is used to sieve out the most important inducing factors as the inputs in E-ELM. C.S. Leung et al. adopt an analog neural network technique, namely Lagrange programming neural network (LPNN), to locate a mobile source. How to find the location of a mobile source from a number of separated sensors is an important problem in global positioning systems and wireless sensor networks. Q.G. Meng et al. propose a way of adapting robot dancing to human preferences by capturing the desired and undesired preferences of human partners and applying them using interactive reinforcement learning (IRL). J. Zhang et al. propose an improved method (IM) to calculate the phase-locking value (PLV) based on the Hilbert-Huang transform. Instead of the Hilbert transform, the Hilbert-Huang transform is used to process non-stationary electroencephalography (EEG) signals. L. Gál et al. present a general view of recent developments in gas sensors based on grapheme unique properties and a new idea of grapheme-based gas $\left(\mathrm{CO}_{2}\right)$ sensor based on the conductance variation. J.C. Wen et al. study the problem of underdetermined blind source separation with the non-strictly sparse condition. A new and more effective algorithm is investigated to estimate the mixing matrices with noise output data sets.

In summary, all of these 19 selected papers presented recent developments of neural-network-related research, with a focus on information processing, modeling, and control using computational intelligence. These papers presented the latest researches in a coherent way. In this editorial, we artificially categorize them into the above three sections to help readers to understand the organization of this special issue.

We hope the reader will share our joy and find this special issue very useful.

Acknowledgments The editors would like to express deepest gratitude to many reviewers who have critically evaluated the papers in several rounds of the review process for this special issue. Their knowledge and professional comments guaranteed the high qualify of the selected papers. In addition, we would also like to thank Dr. John MacIntyre, Editor-In-Chief of the journal Neural Computing and Application, for his great help and suggestions in this process. This publication was made possible by NPRP Grant \#NPRP 4-1162-1-181 from the Qatar National Research Fund (a member of Qatar Foundation). The statements made herein are solely the responsibility of the author[s]. 